\title{
Nuclear morphometric study of prostatic atrophy
}

"Faculty of Medicine, "Ovidius" University, Constanta

${ }^{2}$ Clinical Service of Pathology, Saint Apostle Andrew Emergency County Hospital, Constanta

\begin{abstract}
The prostatic atrophy is a lesion with small glands that can mimic adenocarcinoma, commonly diagnosed in the elderly and whose preneoplastic role is controversial. Over time several classifications have been developed for this lesions according to its architectural and cellular aspects.

Materials and Methods: Aim of the study is morphometrically assessment of epithelial component nuclei in prostatic atrophy. We evaluated parameters represented by nuclear area (NA), nuclear perimeter $\mathrm{P}$ $(\mu \mathrm{m})$ Elongation factor (E), average diameter (DEQ), maximum diameter (Dmax), Minim diameter (Dmin). The control group was represented by adenomatous hyperplasia component of benign hyperplasia of the prostate.

Results: Morphometric analysis revealed significantly higher values of nuclear parameters in atrophy compared with adenomatous component in controls ( $p$ $<0.05$ ) which can signify nucleomegaly and a potential preneoplastic role.
\end{abstract}

Keywords: prostate, atrophy, morphometry, benign hyperplasia

\section{Manuela Enciu}

Faculty of Medicine, "Ovidius" University of Constanta, Mamaia Boulevard, no. 124 University Avenue no 1;

Clinical Service of Pathology, Saint Apostle Andrew Emergency County Hospital, 145 Tomis Avenue, 900591

Constanta, Romania

$+40767745497$

iftimemanuela@yahoo.com

\section{Introduction}

Prostatic atrophy is a benign lesion which in medical practice may raise problems of differential diagnosis with adenocarcinoma, on imaging and from histopathological point of view [1,2]. In some situations, it is necessary to differentiate malignant neoplastic glands from a prostatic atrophy. Also, according to some authors simply focal atrophy or hyperplasia in association with postatrophic inflammation are precursor lesions of high-grade intraepithelial neoplasia and prostate carcinom $[3,4,5,6,7,8]$.

Cytological details such as the shape and size of the nucleus can be quantified using quantitative morphometry, a method are commonly used in research. In this regard, the number of morphometric studies of human prostatic atrophy is reduced internationally. Nuclear morphometry method was introduced in 1982, by Diamond, aiming prognosis of prostate cancer. Due to predictive role in the progression of several types of malignant neoplasms, morphometry provides an outstanding contribution in the clinic and in experimental oncology [9]. Among the assessed parameters, nuclear area is a predictive factor of survival [10]. 
Have been included in the study 20 cases diagnosed with prostate atrophy in men with ages between 55 and 80 . Among the cases presented, 7 cases associated nonspecific chronic inflammation while the rest associated benign hyperplasia. The control group was represented by 20 cases of benign prostatic hyperplasia, adenomatous component, because it is a benign lesion without malignant potential proved. The prostatic tissue was excised by transurethral resection. Histopathology exam and nuclear measurements were performed in the Clinical Service of Pathology, Saint Apostle Andrew Emergency County Hospital of Constanta. The fragments were fixed in 10\% formalin, included in paraffin, sectioned and stained with hematoxylineosin.

For a detailed evaluation of nuclei in epithelial component, we used the semiautomatic morphometry method. Measurements were taken using Nikon microscope equipped with digital camera Nikon image pickup DN 100 pixel resolution of 1280 to 1024, connected to the computer morphometry program Lucia Imaging Laboratory Net - Net Eclipse Software. Have been measured 100 nuclei, 10 nuclei by 10 different fields. Nuclear contour was outlined at a magnification of 400. Previously, the software was calibrated and unit of measurement established was $\mu \mathrm{m}$.

Values thus obtained were registered by the computer program into tables with calculating the average and standard deviation for each parameter. The parameters assessed were represented by: nuclear area (NA) measured in $\mu \mathrm{m} 2$ and standard deviation (dst. NA), nuclear perimeter $\mathrm{P}(\mu \mathrm{m})$ and standard deviation (dst. P), elongation factor and the standard deviation (E and dst. E), average diameter (DEQ), maximum diameter (Dmax) and standard deviation (dst. Dmax), minin diameter (Dmin) and standard deviation of the minimum diameter (Dmin dst).

Statistical analysis of data were performed using Microsoft Office Excel 2007 and statistical software MedCalc for Windows healthcare. Results were considered statistically significant at a $p$ value less than 0.05 . In order to establish correlations between morphometric parameters, we determined the Pearson correlation coefficient (r), which ranging between $(-1,+1)$. Values close to \pm 1 signify a strong correlation, while values close to 0 reflects a weak correlation.

\section{Results}

In terms of histopathology, atrophic lesions were represented by 10 cases of simple atrophy, 7 cases of postatrophic hyperplasia and 3 cases of partial atrophy. Foci of simple atrophy are represented by glands with lobular architecture, sometimes cystic dilated with a basophil aspect of epithelial cells. Lobular or postatrophic hyperplasia is characterized by small size acini, some of them crowded with basophil glands at low magnification and larger nuclei.

The cases of partial atrophy showed diffuse or lobular architecture with crowded glands, eosinophilic cytoplasm and nuclei with slightly increased volume. Values obtained following morphometric analysis of the cases diagnosed with atrophy were summarized in Table I.

Evaluation of nuclear morphometric parameters from the prostatic epithelial component of control Table I - Morphometric values of epithelial cells nuclei in the prostatic atrophy

\begin{tabular}{|l|l|l|l|l|l|l|}
\hline Nuclear parameters in atrophy & $\mathrm{NA}\left(\mu \mathrm{m}^{2}\right)$ & $\mathrm{DEQ}(\mu \mathrm{m})$ & $\mathrm{Dmax}(\mu \mathrm{m})$ & $\mathrm{Dmin}(\mu \mathrm{m})$ & $\mathrm{E}$ & $\mathrm{P}(\mu \mathrm{m})$ \\
\hline Minimal value & 43.38 & 7.43 & 8.24 & 5.26 & 1.2 & 23.19 \\
\hline Maximum value & 65.54 & 9.09 & 13.05 & 8.16 & 1.69 & 29.13 \\
\hline Average & 54.02 & 8.25 & 10.26 & 6.82 & 1.41 & 25.84 \\
\hline Standard deviation & 6.42 & 0.48 & 1.21 & 0.6 & 0.13 & 1.74 \\
\hline
\end{tabular}


Table II - Nuclear morphometric values in controls

\begin{tabular}{|l|l|l|l|l|l|l|}
\hline Nuclear parameters & $\mathrm{NA}\left(\mu \mathrm{m}^{2}\right)$ & $\mathrm{DEQ}(\mu \mathrm{m})$ & Dmax $(\mu \mathrm{m})$ & Dmin $(\mu \mathrm{m})$ & $\mathrm{E}$ & $\mathrm{P}(\mu \mathrm{m})$ \\
\hline Minimal value & 35.83 & 6.75 & 7.55 & 4.81 & 1.1 & 20.22 \\
\hline Maximum value & 56.64 & 8.63 & 11.67 & 8.24 & 1.78 & 27.84 \\
\hline Average & 49.38 & 7.91 & 9.32 & 6.74 & 1.32 & 24.02 \\
\hline Standard deviation & 5.7 & 0.47 & 0.87 & 0.81 & 0.16 & 1.71 \\
\hline
\end{tabular}

cases revealed values in Table II.

Calculation of Pearson correlation coefficient

between the parameters describing the nuclei of prostate atrophy, highlighted a strong correlation between NA and $\mathrm{P}(\mathrm{r}=0.8589 \mathrm{p}<0.0005)$, NA and DEQ $(r=0.9988 \mathrm{p}<0,0001)$, NA and Dmax $(r=$ $0.612 \mathrm{p}=0.0054), \mathrm{AN}$ and Dmin $(\mathrm{r}=0.4822 \mathrm{p}=$ $0.0365)$. The only factor that did not correlate with nuclear area was elongation $(r=-0.04802 p=0.8452)$.

The application of student test (t-test) revealed that between nuclear parameters of atrophy and control group, the differences are statistically significant $(\mathrm{p}<0.05)$, except the minimum diameter.

Calculation of the Pearson correlation coefficient (MedCalc) between morphometric parameters highlighted that there is a strong correlation between NA and $\mathrm{P}(\mathrm{r}=0.8560, \mathrm{p}<0.0001)$, NA and DEQ $(r=0,9994, p<0.0001)$, NA and Dmax $(r=$ 0.5882, $\mathrm{p}=0.0006)$, NA and Dmin $(r=0.6498, \mathrm{p}=$ 0.0001 ). The only parameter that do not relate with the area's nuclear elongation factor, which presents a type of weak negative correlation $(r=-0.2364)$, but not statistically significant $(\mathrm{p}=0.2364)$.

\section{Discussion}

Although it was regarded a disease of the elderly, it was noticed that occur in young people aged between 19 and 29 years [11].

In some reviews of prostatic pathology, atrophy was a lesion in needle biopsies sometimes over diagnosed as adenocarcinoma [12].

Among all forms of atrophy, partial atrophy mimic frequently prostate adenocarcinoma. It is characterized by crowded glands, eosinophilic cytoplasm of the cells and spaced nuclei.

In large power examination, nuclei generally had a relatively benign aspect, without obviously nucleoli although large nuclei with visible nucleoli were described. In atrophy can be identified atypical nuclei, although they are not as large and eosinophilic as those from adenocarcinoma.

Although prominent nuclei are found in atrophic glands associated inflammation, prominent nucleoli were observed in atrophy without the presence of inflammation. On the other hand, inflammation associated with atrophy can lead to the occurrence of nuclear atypia. Thus, if such an atypical feature is present, reduced quantities of cytoplasm should suggest a lesion that mimics adenocarcinoma and should be viewed with caution [13].

We found that the atrophy parameters values are slightly higher than those of benign hyperplasia. Such an assay certify that in the process of atrophy nuclei are hyperchromic and increase in volume in parallel with decrease of cytoplasm.

Following morphometric measurements we found that increasing of the nuclear area and other parameters that characterize nucleus occurs in parallel. The results are similar to those in specialized literature, according to which the cells are small with basophilic aspect, increased nucleo-cytoplasmic ratio, but the nuclei are uniform without irregularities of the nuclear membrane [14].

Although we can see chromocenters, prominent nucleoli are absent. Usually there are two layers of cells in the acini, but basal cell layer is sometimes difficult to distinguish because of the secretory cell atrophy. In such cases, immunohistochemical stains for high molecular weight citokeratin or other basal cell markers may be useful in highlighting the benign nature of the lesion [15]. 


\section{Conclusion}

In atrophy, nuclear area was higher compared with controls and its growth was correlated with increasing of other parameters. The results were statistically significant. Elevated nuclear parameters in atrophy compared with control cases represented by of adenomatous component of benign hyperplasia of the prostate signify a potential preneoplastic role of atrophy, that remains to be elucidated.

\section{Acknowledgements}

This work benefited from financial support through the project,, CERO-CAREER PROFILE: Romanian researchers, contract no. POSDRU/159/1.5/S/135760, a project co-financed by European Social Fund through the Sectoral Operational Programme Human Resources Development 2007-2013.

\section{References}

1. Herawi, M., Parwani, A.V., Irie, J. \& Epstein, J.I. (2005). Small glandular proliferations on needle biopsies: most common benign mimickers of prostatic adenocarcinoma sent in for expert second opinion. Am J Surg Pathol. 29, 874-880

2. Prando, A. \& Billis, A. (2009). Focal prostatic atrophy: mimicry of prostatic cancer on TRUS and 3D-MRSI studies. Abdom Imaging. 34, 271275

3. De Marzo, A.M., Marchi, V.L., Epstein, J.I. \& Nelson, W.G. (1999). Proliferative inflammatory atrophy of the prostate: implications for prostatic carcinogenesis. Am J Pathol. 155, 1985-92

4. van Leenders, G.J., Gage, W.R., Hicks, J.L., van Balken, B., Aalders, T.W. \& Schalken, J.A. et al. (2003). Intermediate cells in human prostate epithelium are enriched in proliferative inflammatory atrophy. Am J Pathol. 162, 152937

5. Putzi, M.J. \& De Marzo, A.M. (2000). Morphologic transitions between proliferative inflammatory atrophy and highgrade prostatic intraepithelial neoplasia. Urology. 56, 828-32

6. De Marzo, A.M., Meeker, A.K., Zha, S., Luo, J., Nakayama, M. \& Platz, E.A. et al. (2003). Human prostate cancer precursors and pathobiology. Urology. 62(5 Suppl. 1), 55-62

7. Wang, W., Bergh, A. \& Damber, J.E. (2009). Morphological transition of proliferative inflammatory atrophy to high grade intraepithelial neoplasia and cancer in human prostate. Prostate. $69,1378-86$

8. Billis, A. (2010). Prostatic atrophy. Clinicopathological significance. Int Braz J Urol. 36(4), 401-9

9. Veltri, R.W., Partin, A.W. \& Miller, M.C. (2000). Quantitative nuclear grade (QNG): a new image analysis-based biomarker of clinically relevant nuclear structure alterations. J Cell Biochem Suppl. Suppl 35, 151-7

10. Montironi, R. (2001). Prognostic factors in prostate cancer. BMJ. 322, 378-379

11. Gardner, W.A. Jr. \& Culberson, D.E. (1987). Atrophy and proliferation in the young adult prostate. J Urol. 137, 53-56

12. Epstein, J.I., Walsh, P.C. \& Sanfilippo, F. (1996). Clinical and cost impact of second-opinion pathology. Review of prostate biopsies prior to radical prostatectomy. Am J Surg Pathol. 20, 851-857

13. Epstein, J.I. \& Netto, G.J. (2008). Biopsy Interpretation of the prostate. Fourth edition Lippincott Williams \& Wilkins.

14. Srigley, J.R. (2004). Benign mimickers of prostatic adenocarcinoma. Modern Pathology. 17, 328-348

15. Hedrick, L. \& Epstein, J.I. (1989). Use of keratin 903 as an adjunct in the diagnosis of prostate carcinoma. Am J Surg Pathol. 13, 389-396 